\title{
A Cultural Re-mediation Model for Storytelling in Pre-school Education
}

\author{
http://dx.doi.org/10.3991/ijet.v10i7.4623 \\ Nicola Capuano ${ }^{1}$, Angelo Gaeta ${ }^{1}$, Matteo Gaeta ${ }^{1}$, Giuseppina Rita Mangione ${ }^{2}$, Anna Pierri ${ }^{1}$ \\ ${ }^{1}$ University of Salerno, Italy \\ ${ }^{2}$ National Institute of Educational Documentation, Innovation and Research, Naples, Italy
}

\begin{abstract}
The use of the emotional language of stories and the amplification of the empathic driver thanks to the identification with story characters, makes the storytelling a valuable educational approach, especially for children. In accordance with embodied and situated cognition theories, manipulative storytelling proposes interactive environments where it is also possible for learners to manipulate the story through objects and tangible interfaces. In line with this vision, we propose in this paper a new model enabling the design and the execution of educational stories for children aged from 3 to 6 . Stories are seen as sequences of missions: game experiences where children can interact to reach the educational objective. A re-mediation strategy, able to adapt the story on three different axis (immediacyhypermediation, similarity-dissimilarity and aggregationdisaggregation) on the basis of assessment results, is also presented. A proof of concept based on the popular Brother Grimm's Hansel and Gretel tale is then discussed to demonstrate the capabilities of the model in the construction and deconstruction of the building blocks of a story.
\end{abstract}

Index Terms-Re-mediation; Digital Storytelling; Augmented Narrative; Embodied and Situated cognition.

\section{INTRODUCTION}

Embodied and Situated Cognition theories [1] [2] see a learning event as a transformative process that is both contextualized (i.e. occurring in a specific environment) and somatic (i.e. related to body and movements). These theories emphasize the fact that learning happens in a social and cultural context where the learner interacts with objects, technology and cultural substrates [3].

The Montessori Method [4], giving emphasis to the action and manipulation, in some way anticipated the key concepts of such theories. This method has been familiar for several years but recently it is gaining interests in the cultural context also because it fits well with narrative approaches based on storytelling.

Storytelling [5] is largely suitable for the development of both cognitive abilities and knowledge in action [6], supporting the process of meaning construction by means of guided strategies that stimulate discovery. Storytelling merges the learning and emotional dimensions together, creating environments where children (especially in the pre-primary curriculum) can develop skills and knowledge, with special attention paid to the improvement of linguistic abilities and the creation of connections [7].

Today, the most suitable technological environment to deal with such approaches seems to be mobile devices. Vendors are recently extending their offer to pre-school children with solid devices, simple interfaces, educational applications and security tools (e.g. parental control). Touch-sensitive screens can drastically simplify interaction with stories where children are required to interact to achieve a goal. Moreover, the availability of new humancomputer interfaces, based on voice, writing and gesture recognition is capable of further improving educational experiences conveyed by such devices.

In this paper we present a new model that, taking into account the key concepts of the Embodied and Situation Cognition theories (focusing, in particular, on those also included in the Montessori Method), supports the design and the execution of educational stories especially thought to exploit modern developments in mobile technologies.

The stories are delivered as missions i.e. narrative game experiences that children play to achieve a specific goal planned by the teacher. During the mission, children can interact following some predefined actions (e.g. talk, touch, look at, assembly, etc.). Moreover, the missions are grounded on active and creative teaching methods like problem-solving, role-play game, etc.

The defined model is based on the combination of results coming from two research projects, namely FIBAC "Innovative Fruition of Artistic and Cultural Assets" [8] and Inf@nzia DigiTales 3.6, both co-funded by the Italian Ministry of Education, University and Research.

$F I B A C$ is aimed at the valorization of cultural assets in real and virtual museums through cultural re-mediation [9]. The project is developing a virtual guide that, applying techniques based on knowledge and creativity, enables the generation of knowledge paths that add new meaning to cultural resources. Through such paths, the real museum space is re-visited and its role as producer of learning and knowledge opportunities for people is enhanced.

$F I B A C$ results have been updated and contextualized to the educational domain, for children aged from 3 to 6 , in Inf@nzia DigiTales 3.6, whose aim is to promote the use of ICT technologies (social media, open data, cloud, etc.) and the latest human-computer interaction paradigms (e.g. augmented reality, RFID/NFC, handwriting and speech recognition, motion detection, etc.) to define educational practices able to enhance curricular activities and facilitate skills and knowledge acquisition processes.

The paper is organized as follows. After a brief discussion on related work in Section II, the section III introduces the cultural re-mediation methodology defined in $F I B A C$. Section IV describes the extensions defined withinInf@nzia DigiTales 3.6 that considers the key concepts of Montessori pedagogies. Section V presents the whole storytelling model based on the defined theoretical com- 
ponents while Section VI shows a case study, based on the Hansel and Gretel tale. Eventually, section V presents our conclusions and gives insight on future developments.

\section{BACKGROUND AND RELATED WORK}

In the research field of Early Childhood Education there is a wide scientific debate on the genesis of cognitive development. Despite that, from Piaget [10], which had stressed the importance of the interaction with the external environment from the earliest stages of a child's life, to Papert, who believes the learning process a construction of more or less correct and functional representations of the world, the concept of Cognitive Artifacts, i.e. objects and devices that facilitate the development of specific learning, emerges.

The Bruner's contribution [11] focuses on action, image and language as forms of representation of the narration. In this view, the narration is an interpretive and cognitive device: narrative thinking allows you to organize the experience and build shared meanings. Childhood is the phase in which one can profitably exploit narrative approaches for education. In this sense the storytelling has an enormous potential thanks to the use of the emotional language of stories, the sensitization of the child to a contextualized knowledge (based on clues and details), the amplification of the empathic driver and the identification with story characters [12].

The adoption of active learning models since preschool can take advantage of new educational technologies and digitization of content. Narrative environments, mediated by technologies, have helped to improve the potential of linear narrative introducing dynamism, reticularity and different perspectives, approaching the child's cognitive thinking and promoting the understanding of the others and of the oneself in the world [13].

In addition, the use of multiple codes and formats of storytelling (oral, written or visual) and its hyper-mediated or immediate representation, allows the child to continue and complement the development of literacy, composition, expressive, technological and analytical skills [14]. The digital story, on the one hand, integrates four brainframes (alphabetical, video, computational and cybernetics) and, on the other hand, favors multiple and different methods of access to knowledge, stimulating distinct dominances and intelligences.

In response to criticism about the gap that normally preschool narratives would create between good and poor comprehenders (children with good or bad reading skills), the digital storytelling allows to overcome this issue with interactive resources able to stimulate and motivate the child through a direct approach with content, calling him to intervene in the construction and deconstruction of the scenes. Proposed activities can indeed act positively on the ability of children in bridging inferences that allow to build links between textual elements [15].

We can currently distinguish two different teaching trends based on digital storytelling: the Trans-media and the Manipulative, both considered in the proposed model. The Trans-media Storytelling is the process in which the elements that make up a narrative are systematically dispersed across various platforms, in order to create a uni- fied and coordinated entertainment experience. Ideally, each medium provides its original contribution to the unfolding of the story [16].

Different types of representations are possible along the continuum of mediation, ranging from hyper-mediation to immediacy. They can be managed by the story to create different types of immersion like Immersive Fiction (i.e. "engagement of the imagination in the mental construction and contemplation of a story world") based on hypermediation, and Immersive Play (i.e. "deep absorption in the performance of a task") based on immediacy [17]. In both cases the medial component supports the creation of a Augmented Narrative that improves traditional narrative pedagogy.

The concept of Augmented Narrative finds its highest expression in a second continuum that can be defined between construction and deconstruction of digital contexts. This continuum has its foundations in active pedagogy and particularly in studies of Froebel (Froebelinspired Manipulatives or FiMs) on the importance of manipulating objects, subsequently taken to be applied to abstract concepts from Montessori (Montessori-inspired Manipulatives or MiMs) [18].

In recent years, the purely playful component of the digital manipulatives has been associated with narratology giving birth to the so-called Manipulative Storytelling. The child is no longer limited to manipulate the object (a toy) but the object itself is introduced and becomes part of an articulated story. StoryToy [19], Magic Story Cube [20] and Reactoon System [21] are examples of interactive storytelling environments where the narration is associated with the possibility to manipulate the story through objects and tangible interfaces.

The manipulation reinforces the levels of interactivity and immersion given by the medial continuum improving at the same time the imagination process, crucial for the child's learning. The integration between mediation and manipulative components creates a different tale in which the degree of involvement and learning is stressed and further strengthened by both the playful and exploratory components.

The model described in the following sections goes exactly in this direction and enables the design and the execution of educational stories based on Trans-media and Manipulative Storytelling approaches.

\section{The Re-Mediation Methodology}

As seen in the section II, the convergence of different media [22] is the core of Trans-media Storytelling. FIBAC proposes to integrate new perspectives in this view, based on the convergence of different types of knowledge, (i.e. factual and social), and on the value of cross-fertilization among domains, (i.e. cultural and not cultural). A cultural resource is so re-mediated not only with respect to media but mainly with respect to its meaning.

Figure 1 gives an overview of the defined methodology for cultural remediation, applied to a conceptual scenario related to a sample cultural resource: the famous Leonardo da Vinci's painting Virgin of the Rocks. The methodology leverages on knowledge and ontology matching techniques described in [23] and [24]. 
At the left-hand side of the figure there is a conceptual scenario related to the Virgin of the Rocks while at the right-hand side, two possible re-mediated scenarios are depicted. The conceptual scenario presents the factual knowledge (e.g. events, actors, time and space) about a cultural resource. The scenario is built basing on domain knowledge about the resource, on knowledge background and preferences of the user, on environment (i.e. real or virtual) and technical (i.e. used devices) contexts.

After the delivery of this scenario, the methodology provides an assessment according to both the cognitive and the emotional perspectives. Emotional approaches are combined with traditional cognitive ones to evaluate both the user engagement and the cognitive growth. In Figure 1, we have oversimplified the assessment results with two labels: I like and I do not like, that can lead to different strategies to build a re-mediated scenario.

Indeed, the assessment results can be a combination of engagement levels and cognitive growth (Figure 2). On the basis of the assessment, the appropriate strategy to adapt the experience in the re-mediated scenario is selected. A re-mediated scenario has the objective of making more engaging the experience delivery by adding new meaning to the resource through additional contents about similar or dissimilar resources.

In case of positive assessment (e.g. high engagement and cognitive growth in Figure 2) we exploit a similarity strategy maintaining the same context of the conceptual scenario. In this case, in general, the objective of the remediated scenario is to provide additional meaning to a resource such to improve the cognitive growth of the user. Additional meaning is offered providing new knowledge on similar topics (e.g. relating knowledge about the Paris and London versions of the Virgin of the Rocks).

The methodology leverages on semantic technologies and inference rules to discover correlations between two resources, in order to assign them additional meaning, not evidenced in the conceptual scenario. We extract a set of features from the contents of the conceptual scenario (in the case of Figure 1 with a feature extraction function we could extract terms such as museum, Leonardo, Virgin of the Rocks, Christ, etc.) and use the extracted features to perform a search in a external dataset like Europeana Linked Open Data or DBPedia.

Once identified the appropriate external datasets, the next step is to identify relevant classes inside the datasets containing matching individuals (e.g. the London version of the same painting). To this purpose we use ontology matching techniques.

In case of negative assessment (e.g. low engagement and cognitive growth in Figure 2), a dissimilarity strategy is exploited to mix the cultural context with a non-cultural one. Analyzing linked data, social network posts and other user generated content, the knowledge about the cultural resources is correlated with the knowledge available on the Web filtered according to the non-cultural interests of the user (e.g. hobbies, sports, music). The idea of connecting different contexts that are not immediately obvious is at the basis of the so-called lateral thinking that we use to provide a new meaning to a cultural resource.

The purpose is twofold: on the one hand, to raise the interest of the user in the cultural resource through the provision of related content matching her/his preferences and,

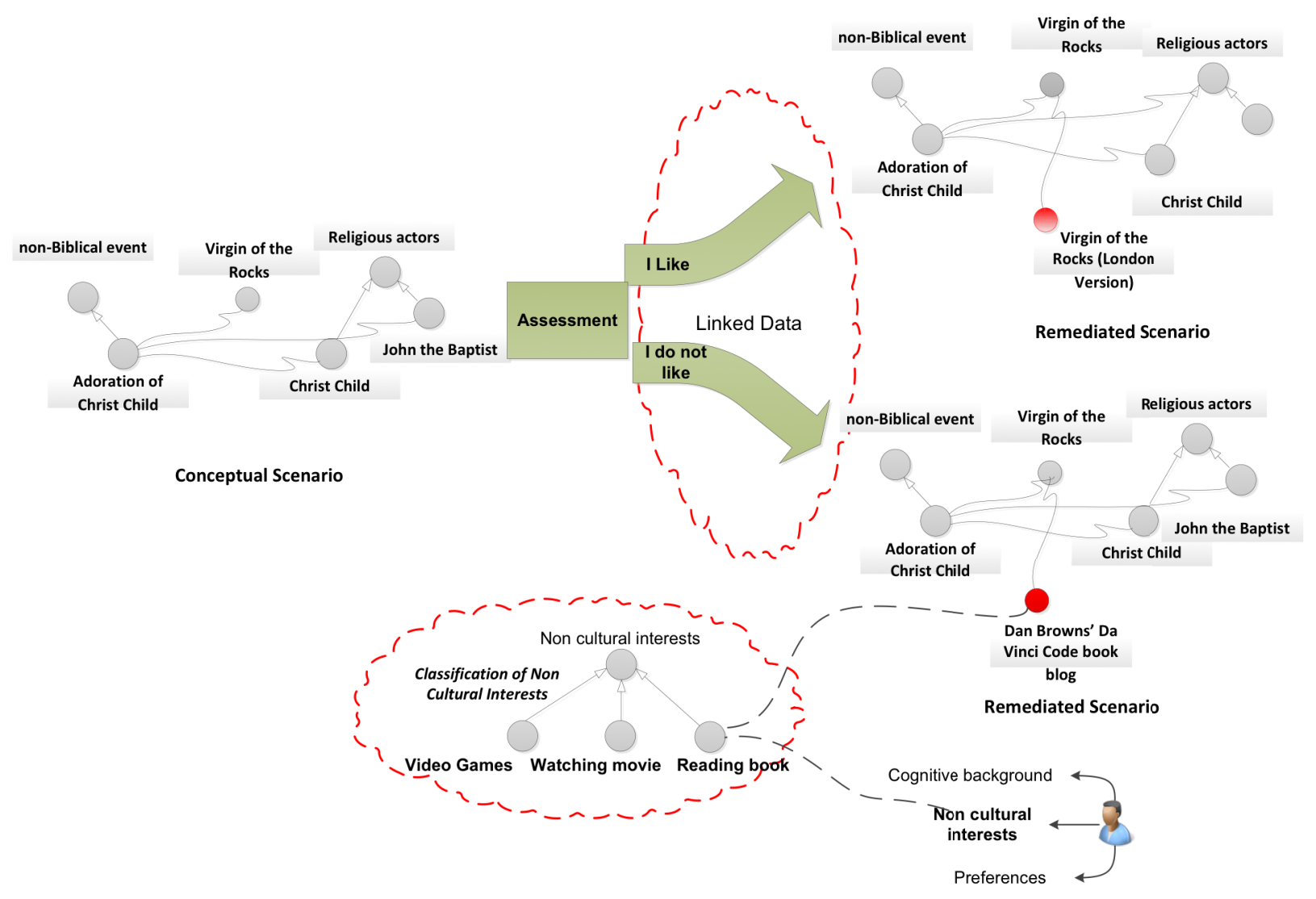

Figure 1. Example application of the cultural re-mediation methodology 


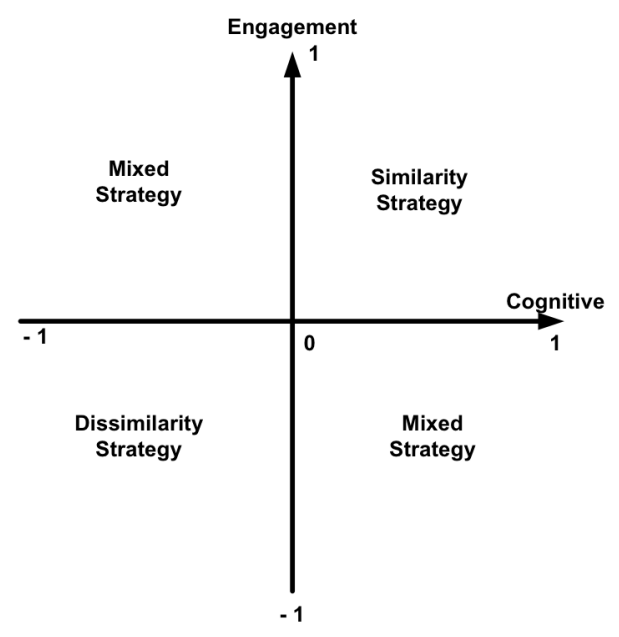

Figure 2. Assessment dimensions and strategies

on the other and, to offer a new and unconventional meaning of a cultural resource by providing contents about noncultural objects (e.g. a description of the Virgin of the Rocks taken from a blog commenting the Dan Brown's famous book Da Vinci's Code).

A side effect of a scenario re-mediated according to a dissimilar strategy is to open the mind of the user offering additional and not obvious perspectives about a cultural resource. To do that, features extracted from the user profile about his/her interests are used to identify external datasets, while feature extracted from the cultural resource are used to identify, in the selected dataset, the appropriate instances. In the case of the example provided in Figure 1, if the user is interested in reading, the first step can result in the identification of the OpenLibrary dataset, while the second one in the identification of the specific blog about the Da Vinci's Code book.

\section{AdDing the Manipulation Perspective}

As anticipated in section I, within Inf@nzia DigiTales 3.6 we improved and extended the methodology described in section III (originally thought for the fruition of cultural assets) to let it feasible for the design and the execution of educational stories targeted to children aged from 3 to 6 . To do that we also relied on results coming from [25].

Conceptually, the methodology described in section III

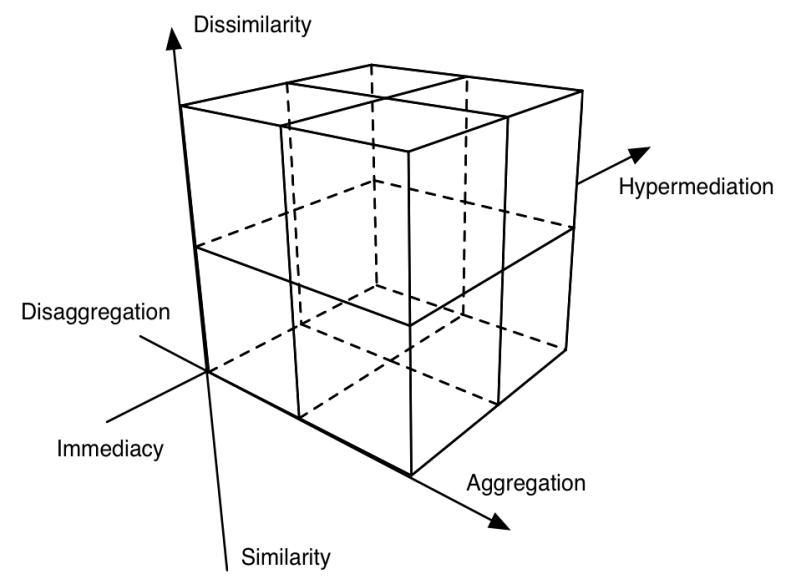

Figure 3. The three re-mediation axis proposes a new approach to media education that is not just focused exclusively on the competition between old and new media (immediacy vs. hyper-mediation) but also strongly considers the knowledge dimension and its acquiring modes through continuity or differences related to prior knowledge (similarity vs. dissimilarity).

In general, immediacy tends to remove the mediated nature of an experience while similarity tends to reduce discontinuity elements of the context and knowledge. Just as hyper-mediation tends to enhance the use of media, dissimilarity tends to maximise the benefits resulting from the connection between heterogeneous contexts and knowledge. Educational and cultural experiences become thus enriched and potentially achievable in order to fulfil students' interests and their learning styles.

To take into account these reflections, we extended the re-mediation methodology taking into consideration a new axis that represents, at the extremes of a continuum, the polarization of the cognitive experience with regard to different models and approaches of active and creative teaching for the 3-6 years age group.

In accordance to the main principles of Manipulative Storytelling, as summarized in section II, we also added a new manipulation axis, meant to enhance children's transformation and creation skills. At the extremes of such axis there are the following modes:

- aggregation, intended as a mode that enhances children's abilities to aggregate contents and minimal elements (e.g. building blocks) of the educational space in order to create new stories and experiences (e.g. to mix the elements of a story in a different way);

- disaggregation, intended as a mode that enhances children's abilities to disjoint a story (or some more complex object), analysing it and identifying its contents and minimal elements.

Manipulation, as a matter of fact, tends to be a functional element of pre-school learning that develops kinaesthetic intelligence and sensory engagement: in other words, it involves children in a natural way of learning that encompasses all senses (e.g. touch, vision, hearing, etc.) in a constructive process [26].

According to this vision, a educational experience will thus be considered as one of the eight cases (octants) determined by the combination of the three polarities identified in Figure 3.

To exemplify, a combination immediacy-similaritydisaggregation represents a mode that leads the child to analyse and disjoint a story or a tale (e.g. The wolf and the seven little goats tale) in its essential elements (e.g. the cheating worked by the wolf knocking at the door) in a continuity of contexts and contents referred to a former story (e.g. Little Red Riding Hood) and reduces the mediated aspects of the experience. In this case, the continuity of the contents related to a previous story (or experience) is a feature of similarity, whereas the reduction or the suppression of the character mediated by experience is a typical feature of immediacy

This re-mediation model is used as the basis for the development of a augmented narrative, as described in section II, by improving narrative pedagogy through elements of immersion, participation, play and handling, organical- 


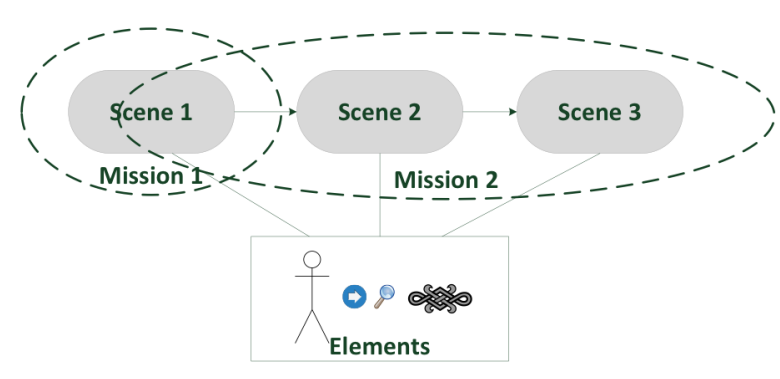

Figure 4. Missions, scenes and elements

ly inserted in missions that are multi-disciplinary and cross-cutting with respect to historical/artistic and cultural perspectives.

\section{THE STORYTELLING MODEL}

Following the re-mediation methodology defined in sections III and IV, a story can be seen as a sequence of missions: a narrative game experience where children can participate and interact in order to reach the educational objective defined by the teacher.

This idea is strongly influenced by the works of Montessori [27], which is based on the compliance to the child's natural psychological development, favoured by the possibility of independent choice among a series of pre-established options and activities to be carried out, thus embracing the constructivist model where learning occurs by means of direct contact and working with objects.

During the mission, children can interact on the basis of predefined actions (i.e. talk, touch, look, assemble). The teacher must introduce children in a magical word, never revealing in advance the mission or the structure of the story. A mission is split in scenes, key parts of a story that are in turn composed of several elements (see Figure 4).

For instance, a mission related to the tale Hop-o '-MyThumb can be "to go home" where the character has to leave stones on the ground in order to find the path for home (scene). After a mission, other missions can be executed following the logical flow of a tale.

Key elements always present in a tale that can be used by digital storytelling are: the hero i.e. the main character, the antagonist i.e. the character who compete with the hero, the helper i.e. the character who supports the protagonist in completing a mission, the location where the tale is set (a castle, a forest, a lake, a mountain, etc.) and the things to do e.g. to save someone, to retrieve something, etc. In the Table I we present the key elements characterizing the two similar tales of Hansel and Gretel (by Brothers Grimm) and Hop-o'-My-Thumb (by Charles Perrault).

\section{CASE STUdy}

In this section we report an example application of our approach for emphasizing the possibilities offered by the model and the underlying re-mediation methodology. The example is centered on the Hansel and Gretel tale. The tale is split in missions with a specific educational objective and each mission consists of one or more scenes. Each scene relates to a specific motif of the tale.

In narrative terminology, a motif is a recurring element that has symbolic significance in a story. Motifs have been classified and indexed in a wide catalogue by Aarne,
Thompson and Uther (ATU) in [28]. A motif that is present in Hansel and Gretel and similar tales (like Hop-o'My-Thumb) is the abandon that usually involves poor parent forced (or persuaded) to abandon children. Besides indexing the scene with regards to the ATU catalogue, an author has also to annotate the mission with respect to the educational objectives that can cover.

For instance, in the frame of the educational objective "raising awareness of the value of food", an author can design a mission containing the scene of the abandon of Hansel and Gretel. In this mission, the poor father is persuaded by the stepmother to abandons his children in the forest and Hansel and Gretel must find a way to return home. This mission aims at stimulating reflections on how the lack of food can generate pain and desperation.

To give an example, an educational experience based on the Hansel and Gretel tale with the objective of raising the awareness of the food in children can be composed by the following three missions.

1. Parents are forced to abandon Hansel and Gretel in the forest since they can not feed them. The objective in this mission is to help Hansel and Gretel in finding a way to return home. This mission aims at stimulating reflections on how the lack of food can generate pain and desperation.

2. Because of hunger, Hansel and Gretel approach the gingerbread house. The children (i.e. the users) in this mission can $(i)$ decompose the gingerbread house by selecting the part of the house they would like to eat, or (ii) build the gingerbread house by aggregating its parts. The reflection we want to stimulate is that food, in some cases, can be a temptation.

3. As mentioned, hunger pushes Hansel and Gretel to approach the gingerbread house, but it is their goodies to lure them in, where they are imprisoned by the old witch who lives in that house and eats children. In this mission, children (the users) eat the part of the house they have previously created (or decomposed). After a certain number of pieces, they are prisoned by the witch. The reflection is to avoid to overdo with food.

When the story ends an assessment aimed at evaluating if children are catching the messages behind the missions, is performed.

In case of negative evaluation, to reinforce the message and re-stimulate reflection, the system proposes alternative missions similar to the one the children didn't understand. With regard to the example of Hansel and Gretel and to the objective of raising the awareness of the food, the three missions present several cases of similarity. The first mission, referring to the lack of food, presents simi-

TABLE I. KEY ELEMENTS IN EXAMPLE STORIES.

\begin{tabular}{|l|l|l|}
\hline \multirow{2}{*}{ Characters } & \multicolumn{2}{|c|}{ Tales } \\
\cline { 2 - 3 } & Hansel and Gretel & Hop-o'-My-Thumb \\
\hline Hero & Hansel & Hop-o'-My-Thumb \\
\hline Antagonist & Witch & Ogre \\
\hline Helper & Gretel & Ogre's wife \\
\hline Location & Gingerbread house & Ogre's castle \\
\hline Things to do & Escape from the witch & $\begin{array}{l}\text { Save himself and his } \\
\text { brothers from the ogre }\end{array}$ \\
\hline
\end{tabular}


larities with Hop-o'-My-Thumb; the second mission presents instead similarities with Snow White (Snow White is in fact tempted by the red apple); the third mission presents similarities with Pinocchio and the land of toys.

In the case of positive assessment, the system proposes different missions aimed at stimulating new and different reflections on the same educational objective. With respect to our example, the missions are purposed at stimulating reflections on some negative aspects of the food, e.g. desperation due to its lack, temptation and issues due to exaggeration. Anyway, food can be also related to positive aspects such as happiness, fertility, power.

To stimulate additional reflections of these facets of food, the system proposes a new mission, dissimilar to the first one, based on the Japanese Momotaro tale. If the motif of the first mission of Hansel and Gretel was the abandon of children, in Momotaro a childless woman and her husband discover a child when they try to open a peach to eat it. If the reflection of the first mission of Hansel and Gretel was that the lack of food causes pain, here an antithetic reflection is stimulated. In the first case there is the abandon of children given the impossibility of the parents to get enough food; in the second case, through a peach, parents adopt a child.

With respect to the presentation of the narrative game, a detailed design of the User Interface (UI) for preschool children is on-going. Following literatures guidelines on this topic, we have to consider the impact of the dichotomy Immediacy-Hypermediation [29] [30]. As an example, the table II (adapted from [31]) presents the main differences among the two modes with respect to the UI.

Designing a UI following the immediacy perspective tends to achieve a realistic experience, where children attention is focused more on the content than on the medium, giving children a feeling of movement and interaction freedom, avoiding the fragmentation of media and contents and reducing as much as possible hotspots activation and recommendations. An example of UI based on the immediacy perspective is shown in Figure 5. It shows the main scene that follows UI design principles (e.g. clear distinction of clickable and non clickable items, one-click interface instead of double clicking, etc.) with a minimum set of additional media to present the story (e.g. the audio guide).

Conversely, designing a UI basing on hypermediation perspective tends to provide a more mediated experience where several media (e.g. audio guides, videos, text) can be added in the main scene, where recommendations and suggestions can suddenly appear and children experience can be fragmented thought different contents. In this case, children have a clear evidence of the interactions with the medium (see Figure 6). Hypermediation mode raises new challenges related to attention management of the children

TABLE II. IMMEDIACY-HYPERMEDIATION IMPACT ON UI.

\begin{tabular}{|l|l|}
\hline \multicolumn{1}{|c|}{ Immediacy } & \multicolumn{1}{c|}{ Hypermediation } \\
\hline $\begin{array}{l}\text { User feels that the medi- } \\
\text { um has been erased }\end{array}$ & $\begin{array}{l}\text { The medium is perceived } \\
\text { by the user and plays a } \\
\text { key role }\end{array}$ \\
\hline Unified perspective & Fragmented perspective \\
\hline $\begin{array}{l}\text { Reality is reached as } \\
\text { much as possible }\end{array}$ & $\begin{array}{l}\text { Experience of the medi- } \\
\text { um is itself an experi- } \\
\text { ence of the reality }\end{array}$ \\
\hline
\end{tabular}

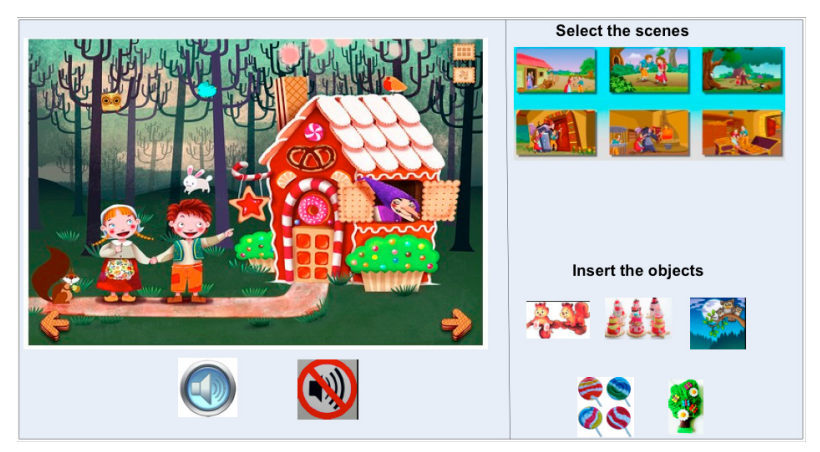

Figure 5. UI based on the Immediacy perspective

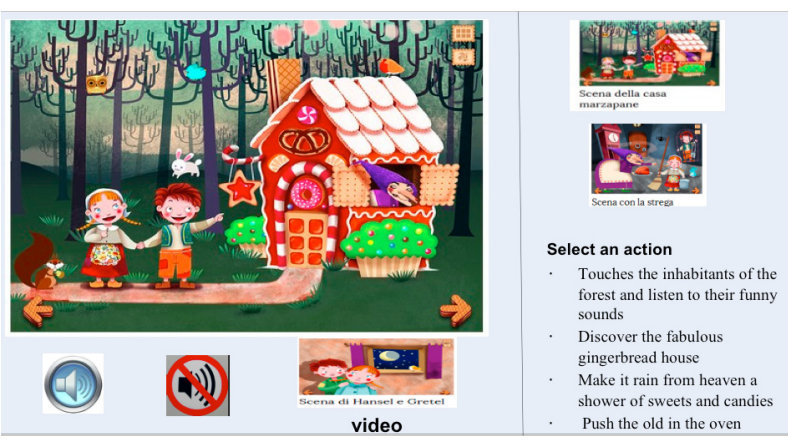

Figure 6. UI based on the Hypermediation perspective

and the UI design must reach a right trade-off between the main scene and the additional media, to avoid that children attention is more focused on interaction than on contents.

The presented case study shows how narrative game experiences can be built following the perspectives of the defined model. Leveraging on the tales paradigm and on the similarity-dissimilarity perspectives, our approach supports also cross-cultural fertilization. The Momataro example in fact can provide children with an additional and different perspective about the food, typical of the Japanese culture.

Furthermore, following the same approach, also the same tale can be presented in its variants to emphasize the different meanings of the same myth. For instance, there are more than 340 variants of the Cinderella tale around the world, each of them providing a different facet of the unjust oppression myth [32].

From a technical viewpoint, the similarity-dissimilarity perspective of the model presents some challenge. Indeed, the search for similar motifs is simplified by several works on motif annotation and on the adoption of topic models [33], the latter useful also to consider, at a certain extent, dissimilar motifs.

In order to consider dissimilarity also regarding crosscultural meaning, a more sophisticated approach working on text corpora is required to understand the different relationships among tales and tale motifs. In this direction goes the adoption, in [34], of the Principal Component Analysis that works on the basis of statistical analysis. We are currently evaluating a different approach based on knowledge extraction techniques, already validated in [35], to extract key concept from text corpora of motifs and semantic correlation techniques to correlate motifs with respect to different perspectives. 


\section{CONCLUSION AND FUTURE WORK}

We presented a re-mediation model that can be applied to define educational stories for children aged from 3 to 6 . The model combines results already obtained on cultural re-mediation with the key concepts of the manipulative strategy behind the Montessori method.

The model is suitable to be implemented with new generation mobile technologies such as handheld devices, tablets and smartphones delivering intuitive and touch interfaces which enhance the senses. This combination is able to support the shift of the learning process form a traditional memory/recall/transfer formula toward the more engaging triple creation/discovery/interconnection through manipulative actions like aggregation and disaggregation of key blocks.

The work around the model is still ongoing and the possibility to include the additional axis of representation, ranging from symbolism to realism is under evaluation. The new axis will support the outline of the stories in order to include, where it might result strategic from a teaching viewpoint, the use of metaphors, allegories, synaesthesia (e.g. psalms, parables, Aesop's fables, etc.) and, on the other hand, the use of a narrative style closer to reality based on free reported speeches, logical axioms, narrative regression (e.g. documentaries, popular chronicles, etc.).

The case study is still in progress and we aim to validate the model in the context of the ongoing project Inf@nzia DigiTales 3.6.

\section{REFERENCES}

[1] F. J. Varela, E. Thompson and E.Rosch. The embodied mind: Cognitive science and human experience. Cambridge, MA, MIT Press 1991.

[2] J. S. Brown, A. Collins and S. Duguid. Situated cognition and the culture of learning. Educational Researcher 18 (1): 32-42, 1989. http://dx.doi.org/10.3102/0013189X018001032

[3] M. Montessori and G. L. Gutek. The Montessori method: the origins of an educational innovation. Rowman \& Littlefield, 2004.

[4] M. L. Anderson. Embodied Cognition, A field guide. Artificial Intelligence, 149, 91-130, 2003. http://dx.doi.org/10.1016/S00043702(03)00054-7

[5] G. R. Mangione, F. Orciuoli, A. Pierri, P. Ritrovato and M. Rosciano. A New Model for Storytelling Complex Learning Objects. Proc. of the $3^{\text {rd }}$ Int. Conf. on Intelligent Networking and Collaborative Systems (INCoS), 836-841, 2011. http://dx.doi.org/10.1109/incos.2011.27

[6] G. R. Mangione, A. Pierri and N.Capuano. Emotion-based digital storytelling for risk education: Empirical evidences from the ALICE project. Int. J. of Continuing Engineering Education and Life-Long Learning, Inderscience, 24 (2), 184-211, 2014

[7] S. Chiu, C.S. Koong and S. H Fan. Designing Preschoolers' Story-Authoring Software: User Requirements and Feedbacks. Proc. of the World Conf. on Educational Multimedia, Hypermedia and Telecommunications, 1309-1314, 2011.

[8] A. Gaeta, M. Gaeta, N. Capuano, A. Pierri and G.R. Mangione. Enhancing Educational Experiences with Remediation Theory: the Case of the FIBAC Project. Proc.of the $6^{\text {th }}$ Int. Conf. on Intelligent Networking and Collaborative Systems (INCoS), 492-497, 2014.

[9] J. D. Bolter and R. Grusin. Re-mediation. Understanding new media. Cambridge, MIT Press, 1999.

[10] J. Piaget. The language and thought of the child. Psychology Press, 1959

[11] I. Grazzani Gavazzi. La competenza narrativa in età evolutiva: approcci classici e nuove ricerche nella prospettiva cognitivoculturale di Bruner. Rivista di psicolinguistica applicata, 3 (2/3), 1000-1017, 2003.
[12] P. Kendeou, P. van den Broek, M.J. White and J. Lynch, Comprehension in preschool and early elementary children: Skill development and strategy interventions. Reading comprehension strategies: Theories, interventions, and technologies, 27-45, 2007.

[13] R. Josselson and A. Lieblich. Making meaning of narratives. Sage Publications, 1999.

[14] M. Carrozzino, C. Evangelista, V. Neri and M. Bergamasco. Interactive Digital Storytelling for Children's Education. IGI Global, 231-252, 2012.

[15] B. Dogan and B. Robin. Educational uses of digital storytelling: Creating digital storytelling contests for K-12 students and teachers. Proc. of the Int. Conf. of the Society for Information Technology and Teacher Education, 633-638, 2009.

[16] H. Jenkins. Convergence culture: Where old and new media collide. NYU press, 2006.

[17] M.L. Ryan. Interactive Narrative, Plot Types, and Interpersonal Relations. Proc. of the Int. Conf. on Interactive Digital Storytelling (ICIDS), Springer-Verlag, 6-13, 2008. http://dx.doi.org/10.1007/978-3-540-89454-4 2

[18] O. Zuckerman, S. Arida and M. Resnick. Extending tangible interfaces for education: digital Montessori-inspired manipulatives In Proc. of the Conference on Human Factors in Computing Systems (SIGCHI), ACM, 859-868, 2005. http://dx.doi.org/10.1145/1054972.1055093

[19] W. Fontijn and P. Mendels. StoryToy: the Interactive Storytelling Toy. Proc. of the $2^{\text {nd }}$ Int. Workshop on Gaming Applications in Pervasive Computing Environments, 2005.

[20] Z. Zhou, A.D. Cheok, J.H. Pan and Y. Li. Magic Story Cube: an interactive tangible interface for storytelling. Proc. of the Int. Conf. on Advances in computer entertainment technology (ACE). ACM, 364-365, 2004. http://dx.doi.org/10.1145/1067343.1067404

[21] A. Alves, R. Lopes, P. Matos, L. Velho and D. Silva. Reactoon: Storytelling in a tangible environment. Proc. of the $3^{\text {rd }}$ Int. Conf. on Digital Game and Intelligent Toy Enhanced Learning (DIGITEL), IEEE,161-165, 2010. http://dx.doi.org/10.1109/ digitel.2010.28

[22] M. McLuhan.Understanding Media. The extensions of man. Mentor, 1964.

[23] A. Gaeta, M. Gaeta, P. Piciocchi, A. Vollero and P. Ritrovato. Exploiting semantic models and techniques to evaluate relevance of human resources in knowledge intensive organizations. Proc. of the $8^{\text {th }}$ Int. Conf. on Signal Image Technology and Internet Based Systems (SITIS), 960-966, 2012. http://dx.doi.org/10.1109/ sitis.2012.142

[24] G. Fenza, V. Loia and S. Senatore. Local semantic context analysis for automatic ontology matching. Proc. of Int. Fuzzy Systems Association World Congress (IFSA), 1315-1320, 2009.

[25] G.R. Mangione, N. Capuano, F. Orciuoli, P. Ritrovato. Disaster Education: a narrative-based approach to support learning, motivation and students' engagement. Journal of e-Learning and Knowledge Society 9 (2), 133-156, 2013.

[26] J. Williams and E.L. Do. Locomotion storytelling: kinesthetic intelligence and tangible objects in storytelling creativity. Creativity \& Cognition, 415-416, 2009.

[27] M. Montessori. The absorbent mind. Holt Paperbacks, 1995.

[28] H. J. Uther. The Types of International Folktales: a Classification and Bibliography Based on the System of Antti Aarne and Stith Thompson, FF Communications, Academia Scientarium Fennica, vol. 1-3, 2004.

[29] Chiasson S. and Gutwin C., "Design Principles for Children's Technology," Proceeding of HCI - TR, 2005

[30] Phadung, M., Suksakulchai, S., \& Kaewprapan, W. (2012). The design framework of interactive storybook support early literacy learning for ethnic minority children. Proceedings from the World Congress on Engineering and Computer Science conference. San Francisco, CA.

[31] Dobson, S. (2009) Remediation. Understanding New Media Revisiting a Classic, Seminar.net - International journal of media, technology and lifelong learning, 5 (2)

[32] Cindarella around the world. http://www.edenvalleyenterprises. org/blhrc/educational/cindtour/world.html, last access 24.02.2015.

[33] H. Jenkins. Fans, blogueros y videojuegos: la cultura de la colaboración. Paidós, 2009. 
[34] F. Karsdorp, P. van Kranenburg, T. Meder, D. Trieschnigg and A. van den Bosch. In search of an appropriate abstraction level for motif annotations. Computational Models of Narrative Workshop (LREC), 2012.

[35] A. Gaeta, M. Gaeta, F. Orciuoli, P. Ritrovato. Managing semantic models for representing intangible enterprise assets: the Aristotele project software architecture. Proc. of the $6^{\text {th }}$ Int. Conf. on Complex, Intelligent, and Software Intensive Systems (CISIS), 10241029, 2012. http://dx.doi.org/10.1109/cisis.2012.167

\section{AUTHORS}

Nicola Capuano is scientific officer at the University of Salerno. His research interest is artificial intelligence and, among its applications, intelligent tutoring systems and knowledge representation. He works as a project manager and research consultant within several research and development projects. He is author of several scientific papers. He is scientific referee for International journals and conferences (e-mail: ncapuano@unisa.it).

Angelo Gaeta is scientific officer at the University of Salerno. His research interests are related to distributed architectures, grid and cloud computing, management of virtual organizations, Semantic Web, knowledge management and representation. He is author of several scientific papers related to the previously mentioned research issues. He has been involved in research activities in several EU co-funded projects of the VI and VII Framework Program (e-mail: agaeta@unisa.it).

Matteo Gaeta is an Associate Professor of Information Processing Systems at the University of Salerno. His research interests include Complex Information Systems
Architecture, Virtual Organization and Grid Computing, Software Engineering, Semantic Web and Computational Intelligence. He is the author of more than 150 scientific papers. He is IEEE Member since 2005. He is editor and reviewer of international conferences, workshops and journals. He is the Scientific Coordinator of several International Research Projects. (e-mail: mgaeta@unisa.it).

Giuseppina Rita Mangione is a chief researcher at the National Institute of Educational Documentation, Innovation and Research (INDIRE). Her main research interests are: technology enhanced learning, adaptive learning, CSCL and micro-adaptivity, metacognitive and selfregulated learning and the application of technologies to education in schools. (e-mail: g.mangione@indire.it).

Anna Pierri is research fellow at the University of Salerno. Her research interests are related to e-learning and mathematics education. She is author of many scientific papers published in international journals, proceedings and books, she was scientific coordinator of several national and international projects, she is member of the Italian association of research in Mathematics Education (e-mail: apierri@unisa.it).

This research is supported by the projects $F I B A C$ "Innovative Fruition of Artistic and Cultural Assets" and Inf@nzia DigiTales 3.6, co-funded by the Italian Ministry of Instruction and Research, under the National Operational Programme for Research and Competitiveness. This article is an extended and modified version of a paper presented at the International Workshop on Adaptive Learning via Interactive, Collaborative and Emotional approaches (ALICE 2014), held on September 10-12, 2014, Salerno, Italy. Submitted 08 April 2015. Published as resubmitted by the authors 05 May 2015. 